\title{
AVALIAÇÃO DO PROGRAMA MAIS MÉDICOS COMO POLÍTICA DE FORTALECIMENTO DA ATENÇÃO BÁSICA
}

\author{
EVALUATION OF THE “MAIS MÉDICOS” (“MORE DOCTORS”) PROGRAM AS \\ A STRENGTHENING POLICY OF PRIMARY HEALTHCARE
}

Silvania Moraes Costa, Tuany Santos Souza, Flavia Rocha Brito, Alba Benemérita Alves Vilela, Adriana Alves Nery, Ismar Eduardo Martins Filho

Universidade Estadual do Sudoeste da Bahia - UESB

\begin{abstract}
The "Mais Médicos" ("More Doctors") Program in Brazil aims to solve the shortage of doctors by providing Brazilian and foreign professionals to work in cities with difficult access and marked socioeconomic vulnerability. This study aims to describe existing researches on the implementation of the "Mais Médicos" (More Doctors) Program on Primary Healthcare. This is a descriptive research, in the form of an integrative review of literature according to data collection in the online databases: BVS and Scielo, with interrelations of Boolean operators and and or. The studies analyzed are: opinion articles, editorials, open space technology and debates, denoting the scarcity of original research carried out in this area. It is perceived that SUS ("Brazilian Unified Health System") needs to develop much more to guarantee the universal right to health, since the population, besides the necessity of more doctors, also needs better perspectives of health and social justice.
\end{abstract}

Key words: Family Health Strategy, Health Policy, Primary Health Care.

\section{Resumo}

O Programa Mais Médicos no Brasil tem como objetivo suprir a carência de médicos através do provimento de profissionais brasileiros $e$ estrangeiros para atuar em municípios de difícil acesso e acentuada vulnerabilidade socioeconômica. Esse estudo objetiva descrever as pesquisas existentes sobre a implantação do Programa Mais Médicos na Atenção Básica. Trata-se de uma pesquisa descritiva, sob a forma de revisão integrativa de literatura mediante coleta de dados nas bases on-line BVS, Scielo e Web of Science, com interrelação dos operadores booleanos and e or. Os estudos encontrados são do tipo artigos de opinião, editoriais, espaço aberto e debates, denotando a escassez de pesquisas originais conduzidas nessa vertente. Percebe-se que o SUS precisa de muito mais para garantir o direito universal à saúde, pois a população além de necessitar de mais médicos, precisa também de melhores perspectivas de saúde e de justiça social.

Palavras chave: Estratégia de Saúde da Família, Política de Saúde, Atenção Primária à Saúde. 


\section{Introdução}

No âmbito da saúde pública, a Atenção Básica caracteriza-se por um conjunto de ações de saúde que tem por objetivo desenvolver uma atenção integral que impacte nos determinantes e condicionantes de saúde das coletividades. Neste contexto, diversas mudanças vêm sendo preconizadas através de ações que visam à reestruturação do Sistema Único de Saúde (SUS), possibilitando assim um importante aumento da abrangência dos serviços, principalmente para os indivíduos que vivem em comunidades remotas que enfrentam expressivas dificuldades para conseguirem este acesso a uma saúde de qualidade $^{1,2}$.

Neste contexto, buscando priorizar a Atenção Básica de acordo com princípios e diretrizes do SUS, o sistema de saúde tem passado por profundas mudanças através de ações que visam a reestruturação do Sistema Único de Saúde (SUS), possibilitando assim um importante aumento do acesso aos serviços. Porém, o que ainda pode-se observar atualmente é que populações que vivem em comunidades remotas e vulneráveis enfrentam importantes dificuldades de acesso aos serviços de saúde ${ }^{2}$.

Além dos desafios enfrentados pela atenção básica como financiamento insuficiente, infraestrutura das Unidades Básicas de Saúde (UBS) inadequada, baixa informatização dos serviços e pouco uso das informações disponíveis para a tomada de decisões na gestão e na atenção à saúde, dificuldades são encontradas para ampliar o acesso e a cobertura da atenção básica e podem ser explicadas não apenas pelas desigualdades geográficas na distribuição dos médicos no território, observadas em diversos países e regiões, mas também, pelo fato da proporção de médicos por habitante ser muito menor que a necessidade da população e do SUS. Esses médicos, por sua vez, estão mal distribuídos no território, de modo que as áreas e populações mais pobres são, proporcionalmente, as que mais sofrem os impactos desta escassez, o que possibilita maiores problemas de saúde, por falta de atendimento e consequentemente de recursos que garantam uma melhoria nas condições de saúde dessas populações ${ }^{3,4}$.

Segundo Póvoa ${ }^{3}$, comparativamente, há um descompasso na densidade de médicos por regiões no país, fazendo com que os grandes centros e cidades com maior possibilidade de desenvolvimento se tornem locais que tenham melhor cobertura destes profissionais. Entretanto, desigualdades geográficas na distribuição de médicos podem ser encontradas em vários países e regiões, levando à necessidade de transformações e busca por diferentes estratégias no sentido de ampliar o acesso a serviços de saúde em áreas vulneráveis, e a reestruturar os sistemas de saúde por meio do fortalecimento da Atenção Básica ${ }^{2}$.

Girardi ${ }^{5}$ salienta que quanto mais precárias forem as condições sociais, econômicas e sanitárias de uma determinada região, maior será a necessidade de médicos e a escassez desse profissional, tornando o mercado de trabalho do médico mais inflacionado, diminuindo assim a capacidade que o município tem em atrair e fixar estes profissionais.

Diante dessa problemática no país, o Governo Federal inicialmente criou, em 2011, o Programa de Valorização dos Profissionais da Atenção Básica (Provab) ${ }^{6}$ para atrair os médicos recém-formados a trabalhar em regiões carentes do Brasil, porém apenas $29 \%$ das vagas abertas foram preenchidas. Sendo assim, foi instituído através da Lei 12.871 do Ministério da Saúde em 22 de outubro de $2013^{7}$ o Programa Mais Médicos, que tem como objetivo principal diminuir a carência de médicos nas regiões prioritárias para o SUS, a fim de reduzir as desigualdades regionais na área da saúde, fortalecendo e ampliando a prestação do serviço da atenção básica aos usuários do SUS em todo território nacional.

Para isso, a lei prevê que ações como a ampliação de vagas e reformas educacionais dos cursos de medicina e residências médicas no país, bem como o provimento emergencial de médicos em áreas vulneráveis, sejam realizadas através de investimentos na melhoria da infraestrutura da rede de saúde, para a concretização dos objetivos propostos pelo Programa Mais Médicos ${ }^{7,8}$. Segundo dados do Ministério da Saúde, 63 milhões de brasileiros já estão sendo beneficiados com a presença dos médicos em 4.058 municípios do Brasil, e estimase que até o final de 2018, serão 70 milhões de brasileiros atendidos pelo Programa Mais Médicos na atenção básica ${ }^{9}$.

Entretanto, há controvérsias e opiniões divididas no país sobre a adoção desse programa como fomento para a reorientação da atenção básica e melhoria das condições de saúde das populações mais desfavorecidas. Por se tratar de um Programa recente, pouco ainda se conduziram pesquisas que mostrem os resultados reais já concebidos no Brasil, inerentes à implantação desta política. Assim, existe a necessidade de estudos que reflitam 
sobre os ganhos e perspectivas, bem como dos potenciais desafios enfrentados pela implementação do Programa Mais Médicos e o impacto deste na população que tem sido exposta aos cuidados desses profissionais.

Diante disso, questiona-se qual o comportamento da literatura em relação aos impactos observados na saúde com a implantação do Programa Mais Médicos? Nesta perspectiva, o objetivo desse trabalho é descrever os estudos existentes acerca da implantação do Programa Mais Médicos e seus impactos na Atenção Básica.

\section{Métodos}

Trata-se de uma pesquisa descritiva, sob a forma de revisão integrativa de literatura, que tem como forma de operacionalização reunir e sintetizar resultados de pesquisas sobre um determinado tema, de forma sistemática e ordenada, contribuindo para o aprofundamento do entendimento sobre o mesmo ${ }^{10}$.

Para a sistematização das etapas foi realizada a identificação do tema, seguida do delineamento de critérios para inclusão e exclusão de estudos, categorização dos tipos de estudos incluídos na revisão integrativa, interpretação dos resultados e apresentação da síntese do conhecimento em um quadro sinóptico.

O levantamento bibliográfico foi realizado nas bases de dados on-line Biblioteca Virtual em Saúde (BVS), Scielo e Web of Science, no período de dezembro de 2015 à fevereiro de 2016, através dos descritores "estratégia de saúde da família", "política de saúde, "atenção primária à saúde", "mais médicos", "programa mais médicos" com interrelação dos operadores booleanos and e or. Utilizou-se também a Lei 12.871 de 22 de Outubro de $2013^{7}$ que institui o Programa Mais Médicos para fundamentação da discussão.

Os critérios de inclusão considerados foram: artigos completos originais ou de revisão disponíveis gratuitamente, artigo de opinião, espaço aberto e editorial que abordassem como tema principal o Programa Mais Médicos. Foram encontrados inicialmente 5 artigos disponíveis no banco de dados da Biblioteca Virtual de Saúde BVS utilizando os descritores "Programa Mais Médicos" AND "Saúde da Família", 15 no Scielo utilizando o descritor "programa mais médicos" e 9 na Web of Science utilizando os descritores Programa Mais Médicos* AND Atenção Básica*.
Realizou-se uma leitura flutuante dos títulos dos estudos encontrados, excluindo-se aqueles que apresentaram duplicidade entre as bases, selecionando-se 13 artigos. Estes, por sua vez, passaram por leitura exaustiva de todo o corpo do texto, a fim de selecionar apenas os que de fato abordavam a temática pesquisada. Sendo assim, a amostra final foi composta por 8 artigos que atendiam aos objetivos e critérios de inclusão, utilizando um recorte temporal de 2013 a 2015.

Por fim, foi realizada a caracterização dos estudos por meio de fichamento e compilação de algumas variáveis observadas: referência do artigo (título, identificação dos autores e do periódico, local e ano de publicação), modalidade do artigo, principais resultados e conclusões, base de dados.

\section{Resultados e discussão}

Os estudos encontrados são do tipo artigos de opinião, editoriais, espaço aberto e debates, denotando a escassez de pesquisas originais conduzidas nessa vertente. Assim, os estudos selecionados para esta revisão integrativa foram categorizados num quadro sinóptico, cujas informações baseiam-se na síntese dos principais resultados e conclusões dos autores que discutiram sobre esse tema, conforme o Quadro 1.

Segundo Garcia et al. ${ }^{11}$, há uma extensa literatura que discute formas alternativas de utilização de recursos para a saúde e seus efeitos sobre índices socioeconômicos, todavia o conhecimento sobre esses impactos referentes ao Programa Mais Médicos ainda tem sido pouco explorado.

Nessa perspectiva foram encontradas na literatura opiniões controversas a respeito da implantação desse programa e a significância estatística desse tipo de intervenção sobre os indicadores de saúde, através da atuação de profissionais estrangeiros na Atenção Básica. Assim, Wilson et al. ${ }^{12}$ fizeram uma avaliação de programas que têm como objetivo levar trabalhadores de saúde a regiões mais desfavorecidas, que geralmente possuem baixos indicadores de saúde, denotando não haver evidências claras acerca do uso de incentivos monetários para estimular a ida do médico a áreas com maior vulnerabilidade social, segundo preconiza a própria política, instituída pela Lei $12.871 / 2013^{7}$. 
Quadro 1: Caracterização dos estudos utilizados na revisão integrativa.

\begin{tabular}{|c|c|c|c|}
\hline $\begin{array}{c}\text { REFERÊNCIA DO } \\
\text { ARTIGO }\end{array}$ & $\begin{array}{l}\text { MODALIDADES } \\
\text { DOS ARTIGOS }\end{array}$ & PRINCIPAIS RESULTADOS E CONCLUSÕES & $\begin{array}{l}\text { BASE DE } \\
\text { DADOS }\end{array}$ \\
\hline $\begin{array}{l}\text { Ribeiro RC. Programa } \\
\text { Mais Médicos }- \text { um } \\
\text { equívoco conceitual. } \\
\text { Ciência e Saúde } \\
\text { Coletiva. } \\
\text { 20(2):421-424. }\end{array}$ & Artigo de Opinião & $\begin{array}{l}\text { O Programa Mais Médicos vem determinando um } \\
\text { ruidoso debate na mídia, reflexo principalmente de um } \\
\text { embate entre o governo e a classe médica, trazendo } \\
\text { um clima de ansiedade e incerteza à população. } \\
\text { Existem inúmeras evidências científicas relevantes e } \\
\text { diretrizes elaboradas para melhorar a saúde da } \\
\text { população, o que falta então é a vontade política para } \\
\text { implementar ações baseadas nessas evidências. O } \\
\text { povo não quer mais médicos, o povo quer é mais } \\
\text { saúde! }\end{array}$ & Scielo \\
\hline $\begin{array}{l}\text { Schanaider A. Mais ou } \\
\text { menos médicos! Rev. } \\
\text { Col. Bras. Cir. 2014; } \\
\text { 41(5): 303-304. }\end{array}$ & Editorial & $\begin{array}{l}\text { Jamais a ampliação numérica de médicos resolverá a } \\
\text { dificuldade de alocação destes no interior do Brasil, } \\
\text { pois a grande maioria, na primeira oportunidade, } \\
\text { buscará a iniciativa privada e os convênios, haja vista } \\
\text { comporem alternativas profissionais mais } \\
\text { compensadoras e capazes de permitir a educação } \\
\text { continuada e o contato com os grandes centros. Seria } \\
\text { mais sensato investir nas universidades públicas, que } \\
\text { ainda congregam um corpo docente de excelência, } \\
\text { recuperar seus hospitais universitários, reforçar o seu } \\
\text { corpo técnico-administrativo, dar-lhes um salário } \\
\text { digno, viabilizando a expansão de vagas nos Cursos de } \\
\text { Medicina. Estamos na contramão da história com o } \\
\text { "Mais Médicos", privilegiando a quantidade e } \\
\text { negligenciando a qualidade. }\end{array}$ & Scielo \\
\hline $\begin{array}{l}\text { Oliveira FP, Vanni T, } \\
\text { Pinto HÁ, Santos JTR, } \\
\text { Figueiredo AM, } \\
\text { Araújo SQ, et al. Mais } \\
\text { Médicos: um } \\
\text { programa brasileiro } \\
\text { em uma perspectiva } \\
\text { internacional. } \\
\text { Interface (Botucatu). } \\
\text { 2015; } 19(54): 623-34 .\end{array}$ & Debates & $\begin{array}{l}\text { Apesar do sucesso parcial de iniciativas anteriores, foi } \\
\text { apenas com o Programa Mais Médicos que a provisão } \\
\text { de médicos em áreas vulneráveis teve a magnitude e a } \\
\text { resposta em tempo adequado para atender a } \\
\text { demanda dos municípios brasileiros. A potência do } \\
\text { Mais Médicos, entre outras, relaciona-se com uma } \\
\text { proposta de mudança que não tem medo de procurar } \\
\text { novos caminhos, que dialoga com experiências } \\
\text { nacionais e internacionais e com todos os sujeitos } \\
\text { envolvidos na ampliação do acesso e da melhoria da } \\
\text { qualidade da atenção básica e do SUS. }\end{array}$ & Scielo \\
\hline $\begin{array}{l}\text { Scheffer M. Programa } \\
\text { Mais Médicos: em } \\
\text { busca de respostas } \\
\text { satisfatórias. } \\
\text { Interface. 2015; } \\
\text { 19(54): 637-40. }\end{array}$ & Debates & $\begin{array}{l}\text { Deve-se repensar a abrangência e a magnitude da } \\
\text { atual política brasileira de alocação de médicos em } \\
\text { regiões vulneráveis e se, de fato, a proposta vigente foi } \\
\text { célere, talvez essa rapidez corresponda exatamente ao } \\
\text { seu caráter emergencial. A meta há de ser mesmo } \\
\text { 'procurar novos caminhos', conciliando a } \\
\text { governabilidade do momento com o desenvolvimento } \\
\text { do estado democrático e a promoção do direito } \\
\text { universal à saúde. Para os gestores públicos, ser } \\
\text { governo e, ao mesmo tempo, homens de estado tem } \\
\text { sido um grande desafio contemporâneo. }\end{array}$ & Scielo \\
\hline 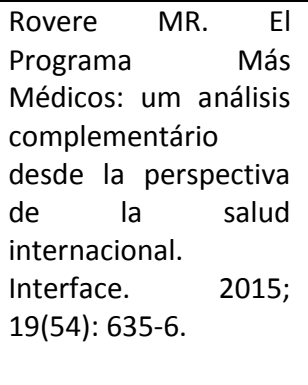 & Debate & $\begin{array}{l}\text { Em todos os casos, não se trata de diferenças ou de } \\
\text { discussões ideológicas nos países, trata-se de saber } \\
\text { como e quando foi que as universidades públicas } \\
\text { deixamos de cumprir os nossos mandatos } \\
\text { institucionais e, sobretudo, qual será a contribuição } \\
\text { que poderemos fazer no futuro para que um programa } \\
\text { como o Mais Médicos não seja necessário, } \\
\text { especialmente numa região onde se aceita que a } \\
\text { saúde é um direito e que o Estado deva garantir esse } \\
\text { direito. }\end{array}$ & Scielo \\
\hline
\end{tabular}




\begin{tabular}{|c|c|c|c|}
\hline $\begin{array}{l}\text { Santos LMP, } \text { Costa } \\
\text { AM, Girardi } r \text { SN. } \\
\text { Programa } \quad \text { Mais } \\
\text { Médicos: uma ação } \\
\text { efetiva para reduzir } \\
\text { iniquidades em } \\
\text { saúde. Ciência e } \\
\text { Saúde Coletiva. 2015; } \\
\text { 20(11): } 3547-3552 .\end{array}$ & Artigo de Opinião & $\begin{array}{l}\text { O Programa Mais Médicos não é um equívoco } \\
\text { conceitual e, além de bem fundamentado em } \\
\text { evidências das necessidades da população, é } \\
\text { concernente aos princípios fundamentais do SUS. O } \\
\text { Programa incide sobre a falta de médicos a curto e } \\
\text { médio prazo. O SUS ainda precisa de muito mais para } \\
\text { garantir o direito universal à saúde, mas as evidências } \\
\text { já indicam melhor distribuição de médicos e maior } \\
\text { oferta de serviços. }\end{array}$ & Scielo \\
\hline 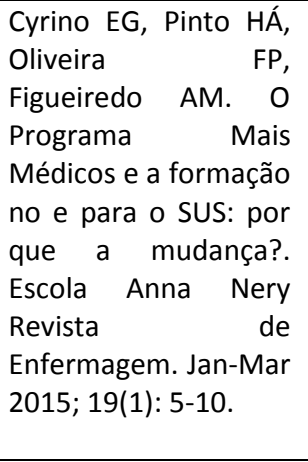 & Editorial & $\begin{array}{l}\text { O debate sobre formação e provimento na saúde, que } \\
\text { valoriza o encontro entre humanos, o compromisso } \\
\text { com a integralidade e a equidade, que responde às } \\
\text { necessidades singulares, à maior aproximação entre } \\
\text { Instituições de Educação Superior e serviço, saúde e } \\
\text { educação, que desencadeia ressignificações, } \\
\text { reconstrução do conhecimento e produção de novos } \\
\text { saberes, num encontro ético, estético e político está } \\
\text { aberto, com a clareza de que abordamos uma prática } \\
\text { social extremamente complexa, que aprendemos a } \\
\text { cada dia, que estamos formando e nos formando e } \\
\text { temos muito para avançar em teoria e prática. }\end{array}$ & Scielo \\
\hline
\end{tabular}

Ao analisar os estudos categorizados no Quadro 1 é possível observar opiniões divergentes sobre a retórica da implantação dessa nova política no país. Ribeiro ${ }^{13}$ discute brevemente sobre os ruídos disseminados pela mídia leiga acerca do assunto, o que segundo o autor, foi criada uma espécie de alarde, quanto à polêmica levantada pela classe médica contra o governo, sugerindo que houve a ocorrência de algumas questões equivocadas na alimentação desse debate.

Esses pontos de debate, por sua vez, são apontados como equívocos de definição do problema, no sentido de delimitar quais são as doenças de maior impacto na população, em função de seu perfil epidemiológico, bem como equívocos nas propostas de enfrentamento a esses problemas, que por sua vez têm reflexo advindos do predomínio de um modelo de saúde alicerçado em políticas públicas dominadas por uma perspectiva médica, curativista, com foco na doença e não nas causas e estilo de vida da população e, por último, no que se refere às propostas desconectadas de evidências científicas relevantes, visto que tanto em políticas consolidadas em outros países, quanto as já existentes no Brasil, tal qual a Política Nacional de Promoção da Saúde ${ }^{14}$ e o Plano de Ações Estratégicas para o Enfrentamento de Doenças Crônicas Não Transmissíveis ${ }^{15}$, recomendam a manutenção da saúde por meio da observação e priorização dos fatores causais e determinantes da saúde, sugerindo que a população necessita de mais saúde e não necessariamente mais médicos ${ }^{13}$.
Tendo em vista a prerrogativa do Governo Federal em abrir a guarda para os médicos estrangeiros baseada na alegação de que a relação de 2 médicos/1000 habitantes afeta o bom atendimento à população, visto que nos EUA essa relação é de 2,7, França 3,4 e Argentina 3,2 , Schanaider ${ }^{16}$ chama a atenção que, dentre outros exemplos, o Chile e o Japão com indicadores de 1,0 e 2,1, respectivamente, possuem uma medicina de alta qualidade, enquanto que o Azerbaijão e Cazaquistão, com indicadores em torno de 3,4 não estão melhores que o Brasil. Isto sugere que o problema não está necessariamente atrelado à quantidade de profissionais por si só, mas sim à qualidade dos serviços que têm sido oferecidos á população, no âmbito da Atenção Primária à Saúde, por meio da Atenção Básica.

Oliveira et al. ${ }^{17}$ analisaram 0 processo nacional e internacional (Austrália e EUA) de fortalecimento da Atenção Básica e provisão de profissionais médicos em áreas vulneráveis e observaram que apesar das diferenças de contexto, a problemática tem semelhanças. Assim, verificaram que o modelo brasileiro e o australiano utilizam combinações de incentivos financeiros e educacionais. Já o modelo estadunidense tem maior foco nos incentivos financeiros e na retenção dos médicos estrangeiros que já estão no país, através do treinamento e da extensão do visto de permanência. Os autores destacam que existem importantes limitações na comparação da atratividade de um país de média renda com países de alta renda, visto que existem muitas 
limitações em relação à possibilidade entre países com propostas políticas extremamente divergentes em relação ao sistema público de saúde.

Neste contexto, Schanaider ${ }^{16}$ e Scheffer ${ }^{18}$ abordam que alguns fatores-chave determinam a escolha, pelo médico, do lugar de seu exercício profissional, influenciando assim a recorrência da polarização destes profissionais em áreas específicas em detrimento de outras. Sabe-se, portanto, que a ausência de atratividade de regiões com piores indicadores sociais e inadequadas condições de trabalho, com má remuneração e cargas horárias excessivas, tendem a dificultar cada vez mais a fixação desses médicos nessas regiões. Assim, a renda elevada pode também não ser um fator preponderante para atração desses médicos, cujo isolamento profissional se torna um agravante, associado a uma baixa qualidade de vida deles mesmo e de seus familiares.

Ainda Rovere ${ }^{19}$ debate sobre o Programa mais Médicos sob a perspectiva da saúde internacional, trazendo diferenças ideológicas como ponto chave na comparação entre os países que não tratem como prioridade um programa como o Mais Médicos, visto que especialmente em regiões onde se institui que a saúde é um direito de todos, o Estado, de fato, deve garantir esse direito. É também nesta perspectiva que outros autores discutem acerca desta política, trazendo a necessidade do Programa Mais Médicos minimizar a carência desses profissionais e reduzir as desigualdades regionais em saúde, envolvendo estratégias que levem em conta a ampliação de vagas e novos cursos de Medicina, baseado numa matriz curricular revisada, bem como investimentos na construção de novas UBS e provimento de médicos brasileiros e estrangeiros, principalmente em áreas de vulnerabilidade, como uma ação efetiva para reduzir iniquidades em saúde ${ }^{8,20}$.

O provimento de médicos, num ponto de vista estatístico ${ }^{21}$ diminuiu o número de municípios com baixa distribuição desses profissionais na Atenção Primária a Saúde, passando de 1.200 municípios em março de 2013, para 558 em setembro de 2014, isto é, uma redução de 53,5\%. Neste sentido, o programa contribuiu para reduzir iniquidades em saúde, uma vez que, na região Norte $91,2 \%$ dos municípios que apresentavam escassez em 2013 foram atendidos, denotando um provimento de aproximadamente 4,9 médicos/município ${ }^{20}$.

Esse, dentre outros argumentos, contrastam a ideia apresentada no estudo de Ribeiro ${ }^{13}$, mostrando que o Programa Mais Médicos não é um equívoco conceitual, embora os médicos estrangeiros tenham tido um processo de formação diferente, o que necessitaria da validação do diploma como prerrogativa para o exercício da profissão, tal qual se procede em outros países. Ainda assim Santos et al. ${ }^{20}$ defende que além de bem fundamentado em evidências de necessidades da população, o programa é concernente aos princípios constitucionais do SUS.

No estudo de Silva et al. ${ }^{22}$ faz-se uma análise do Programa Mais Médicos sob a ótica de estudantes acerca da assistência prestada à uma população indígena, no estado do Tocantins, onde foram abordadas algumas dificuldades dos usuários frente ao atendimento médico. Dentre elas a mais frequente é dificuldade de entender o idioma dos profissionais estrangeiros, bem como dificuldades enfrentadas pelos médicos frente à população, principalmente questões culturais e frente à problemas de infraestrutura, falta de medicamentos e insumos para uso da população, refletindo a necessidade de melhorias no planejamento e gestão dos serviços a serem prestados.

\section{Considerações finais}

Diante da análise dos estudos, percebe-se que as discussões sobre o Programa Mais Médicos divergem consideravelmente, tanto do ponto de vista dos profissionais da classe médica, quanto dos pesquisadores da saúde pública. Percebe-se que o provimento de médicos nas áreas de maior vulnerabilidade social não é a única medida necessária para a melhoria das condições de saúde da população, mas sobretudo é necessário medidas preventivas e conhecimento dos determinantes de saúde e principais causas que levam a população a adoecer.

Além disso, a melhoria das condições de atendimento devem ser preconizados pra que essa política seja consolidada de maneira mais efetiva, através de melhorias na infraestrutura das unidades básicas de saúde, bem como fornecimentos de materiais e insumos para a população.

Devido aos resultados já apresentados pela literatura, espera-se que o programa continue avançando em relação aos índices de saúde, juntamente com o fortalecimento da Atenção Básica. Desta forma, mesmo que algumas evidências já indiquem uma melhor distribuição 
de médicos e maior oferta de serviços, nos territórios remotos, ainda assim o SUS precisa de muito mais para garantir o direito universal à saúde, pois a população além de necessitar de mais médicos, precisa também de melhores perspectivas de saúde e de justiça social.

\section{Referências}

1. Nascimento APS, Santos LF, Carnut L. Atenção primária à saúde via estratégia de saúde da família no Sistema Único de Saúde: introdução aos problemas inerentes à operacionalização de suas ações. J Manag Prim Health Care 2011; 2(1):18-24.

2. Brasil. Ministério da Saúde. Portaria no 2.488, de 21 de Outubro e 2011. Brasília, 2011.

3. Póvoa L, Andrade MV. Distribuição geográfica dos médicos no Brasil: uma análise a partir de um modelo de escolha locacional. Cad. Saúde Pública, Rio de Janeiro. 2006; 22(8):15551564.

4. Grobler L, Marais B, Mabunda SA, Marindi PN, Reuter $\mathrm{H}$, Volmink J. Interventions for increasing the proportion of health professionals practising in rural and other underserved areas. Cochrane Database Syst Rev. 2009; 21(1):1-25.

5. Girardi SN, Carvalho CL, Araújo JF, Farah JM, Wan der Maas L, Campos LAB. Índice de escassez de médicos no Brasil: estudo exploratório no âmbito da Atenção Primária. In:

Pierantoni CR, Dal Poz MR, França T,organizadores. $O$ trabalho em Saúde: abordagens quantitativas e qualitativas. Rio de Janeiro: Cepesc/IMS/UERJ, ObservaRH. 2011: 171-86.

6. Brasil. Portaria Interministerial $\mathrm{n}$ ㅇ 2.087/MS/MEC, de 21 de setembro de 2011. Brasília, 2011.

7. Brasil. Ministério da Saúde. Lei no 12.871, de 22 de outubro de 2013. Institui o Programa Mais Médicos, altera as Leis n. 8.745, de 9 de dezembro de 1993, e n. 6.932, de 7 de julho de 1981, e dá outras providências. Diário Oficial da União. 23 Out 2013.

8. Cyrino EG, Pinto HÁ, Oliveira FP, Figueiredo AM. O Programa Mais Médicos e a formação no e para o SUS: por que a mudança?. Escola Anna Nery Revista de Enfermagem. JanMar 2015; 19(1): 5-10.

9. Brasil. Ministério da Saúde. Secretaria de Gestão do Trabalho e da Educação na Saúde. Programa mais médicos - dois anos: mais saúde para os brasileiros. Brasília, 2015.

10. Mendes KDS, Silveira RCCP, Galvão CM. Revisão integrativa: método de pesquisa para a incorporação de evidências na saúde e na enfermagem. Texto Contexto Enfermagem, Florianópolis. 2008; 17(4): 758-764.

11. Garcia B, Rosa L, Tavares R. Projeto Mais Médicos para o Brasil: Apresentação do Programa e Evidencias Acerca de Seu Sucesso. São Paulo; Informações FIPE; (402): 26-36, mar. 2014. ilus. Disponível em: http://downloads.fipe.org.br/content/downloads /publicacoes/bif/2014/3_bif402.pdf.

12. Wilson NW, CouperID, De Vries E, Reid S, Fish T, Marais BJ. A critical review of interventions to redress the inequitable distribution of healthcare professionals to rural and remote areas. Rural Remote Health. 2009; $9(2): 1060$.

13. Ribeiro RC. Programa Mais Médicos um equívoco conceitual. Ciência e Saúde Coletiva. 2015; 20(2):421-424.

14. Brasil. Ministério da Saúde (MS). Secretaria de Atenção à Saúde . Política nacional de promoção da saúde. Brasília: MS; 2006.

15. Brasil. Ministério da Saúde (MS). Secretaria de Atenção à Saúde . Plano de ações estratégicas para o enfrentamento das doenças crônicas não transmissíveis (DCNT) no Brasil 2011-2022. Brasília: MS; 2006.

16. Schanaider A. Mais ou menos médicos! Rev. Col. Bras. Cir. 2014; 41(5): 303-304.

17. Oliveira FP, Vanni T, Pinto HÁ, Santos JTR, Figueiredo AM, Araújo $S Q$, et al. Mais Médicos: um programa brasileiro em uma perspectiva internacional. Interface (Botucatu). 2015; 19(54): 623-34.

18. Scheffer M. Programa Mais Médicos: em busca de respostas satisfatórias. Interface. 2015; 19(54): 637-40.

19. Rovere MR. El Programa Más Médicos: um análisis complementário desde la perspectiva de la salud internacional. Interface. 2015; 19(54): 635-6.

20. Santos LMP, Costa AM, Girardi SN. Programa Mais Médicos: uma ação efetiva para reduzir iniquidades em saúde. Ciência e Saúde Coletiva. 2015; 20(11): 3547-3552.

21. Estação de Pesquisa de Sinais de Mercado (EPSM). Dados Estatísticos sobre o impacto do Programa Mais Médicos no cenário de escassez de médicos em atenção primária no Brasil. Belo Horizonte, 2015.

22. Silva RP, Barcelos AC, Hirano BQL, Izzo 
RS, Calafat JMS, Soares TO. A experiência de alunos do PET-Saúde com a saúde indígena e o Programa Mais Médicos. Interface. 2015; 19 Supl: 1005-14.

\section{Endereço para Correspondência}

Universidade Estadual do Sudoeste da Bahia UESB

Av. José Moreira Sobrinho, s/n - Jequiezinho Jequié (BA)

CEP.: 45206-510

e-mail: silvaniamoraescosta@hotmail.com

Recebido em 05/01/2017

Aprovado em 25/05/2017

Publicado em 08/07/2017 